(C2016 IEEE. Reprinted, with permission, from A. Alanazi, and K.M. Elleithy, "Energy efficient hidden node detection for improving Quality of Service in wireless multimedia sensor networks.” In Proceedings of 2016 IEEE Long Island Systems, Applications and Technology Conference (LISAT), East Farmingdale, NY, 2016. DOI: 10.1109/LISAT.2016.7494145.

This material is posted here with permission of the IEEE. Such permission of the IEEE does not in any way imply IEEE endorsement of any of the University of Bridgeport's products or services. Internal or personal use of this material is permitted. However, permission to reprint/republish this material for advertising or promotional purposes or for creating new collective works for resale or redistribution must be obtained from the IEEE by writing to pubs-permissions@ieee.org. By choosing to view this document, you agree to all provisions of the copyright laws protecting it. 


\title{
Energy Efficient Hidden Node Detection for Improving Quality of Service in Wireless Multimedia Sensor Networks
}

\author{
Adwan Alanazi and Khaled Elleithy \\ Computer Science and Engineering Department \\ University of Bridgeport, CT- USA \\ aalanazi@my.bridgeport.edu; elleithy@bridgeport.edu
}

\begin{abstract}
In many wireless multimedia sensor networks (WMSNs) the nodes are static. However, node connection is subjected to change due to disruptions in wireless communication, power changes in transmission, or loss of synchronization between neighboring nodes. A sensor should constantly be aware of its immediate neighbors, through a process called continuous neighbor discovery. In this paper we introduce an energy efficient hidden node detection (EEHND) algorithm for continuous neighbor discovery process in the wireless multimedia sensor networks (WMSNs). We focus on the continuous neighbor discovery process and regard it as a combined task of all the nodes in every connected segment. Each sensor is entered as a coordinate in an effort in order to reduce the time to detect hidden sensors. Based on the simulation results, we demonstrated that the protocol detected the hidden nodes in the network.
\end{abstract}

Keywords: wireless multimedia sensor networks, neighbor discovery, hidden nodes, and energy efficiency.

\section{INTRODUCTION}

The latest advances in the complementary metal-oxidesemiconductor (CMOS) technology have led to the development of wireless multimedia sensor networks as a class of wireless sensor network. WMSNs are capable of capturing multimedia content such as images, audio and video about the surrounding environment. Then the WMSNs send that to the sink or a base station. WMSNs have offered numerous applications in surveillance, environmental applications military applications, health applications, and home appellations. However, multimedia applications have limitations that will affect the successful media transmission in the sensor networks. Node connection is subjected to change due to disruptions in wireless communication, power changes in transmission, or loss of synchronization between neighboring nodes [1-4]. When a sensor is aware of its immediate neighbors, it must continuously maintain information on its surroundings. In this work, we differentiate between neighbor discovery during sensor network initialization and continuous neighbor discovery. We focus on the latter and regard it as a combined task of all the nodes in every connected segment. Each sensor initiates the neighbor discovery process to reduce power consumption by not increasing the time required to detect hidden sensors. Despite the static nature of the sensors in many sensor networks, connectivity is still subject to change even after the network has been established. The sensors must continuously try to identify new neighbors in order to fulfill these requirements: 1) Local synchronization loss because of accumulated clock drifts [5]; 2) Wireless connectivity disruption between adjacent nodes by a temporary event, such as a passing vehicle, moving animals, or storms. When these events are over, the hidden nodes must be revived for rediscovery; 3) the ongoing accumulation of new nodes, in some networks to compensate for nodes which have malfunctioned; 4) and the increase in transmission power of some nodes, in response to several events, such as detection of emergency situations [6-8]. Based on these circumstances, detecting new links and nodes in sensor networks must be considered an ongoing process [7]. In the following discussion, we distinguish between the detection of new links and nodes during initialization. When the node is in an active state that performs normal recovery process. The normal recovery process consists of initial neighbor discovery and then continuous discovery process for finding the location of the node. The former is referred to as initial neighbor discovery whereas the latter is referred to as continuous neighbor discovery. While previous works address initial neighbor discovery and continuous neighbor discovery as similar tasks, to be performed by the same scheme, we claim that different schemes are required, based the following reasons:

Initial neighbor discovery is usually conducted when the sensor has no evidence about the structure of its immediate surroundings. In this situation, the sensor cannot communicate with the gateway and is therefore very limited in performing its tasks. The immediate surroundings should be detected as soon as possible in order to set a path to the gateway and contribute to the operation of the network [9]. Hence, in this state, more extensive energy use is justified. In contrast, continuous neighbor discovery is performed when the sensor is already functioning [8]. This is a long-term process, whereby optimization is vital for extending the network lifetime. When the sensor initiates the continuous neighbor discovery, it shows that a node is already responsive from many of its immediate neighbors, and can, therefore, accomplish the task with these neighbors to consume minimum energy $[10,11]$. In contrast, each sensor in a distinct fashion must accomplish an initial neighbor discovery.

The remainder of the paper is organized as follows: Section II discusses the problem identification and its significance. Section III gives an overview of existing approaches. Section IV presents the proposed approach. Section V discusses the 
simulation and experimental results. Finally the entire paper is concluded in Section VI.

\section{PROBLEM IDENTIFICATION}

Initial neighbor discovery is usually performed when the sensor has no awareness of its immediate surroundings. In such a situation, the sensor node cannot communicate with its base station thus, limiting the node performance. Based on this situation, several applications experience the following problems:

- The network experiences heavy traffic; as a result the performance is decreased.

- Extended process for forwarding the data; it takes longer process for forwarding the data if node is not found then it causes the latency in the network.

- Additional energy consumption. When the neighbor node information is not available then the source node starts sending the data so that all the nodes receive the data, consume additional energy.

\section{RELATED WORK}

In this section, some of important features of related work are discussed. In [5], the neighbor discovery process is introduced and attempts to determine the new node from the station. Since, energy consumption is not a concern for the base station, discovering new nodes is simplified. The base station initiates the node discovery process periodically broadcasting the special HELLO message. When a node gets the message, it initiates the registration process. The node can switch channels to find the best HELLO message. The best Hello message might depend on the distinctiveness of the broadcasting base station, on security considerations, or on the physical layer quality. Another related work attempted to reduce neighbor discovery time process by improving the broadcast rate of the HELLO messages $[10,11]$. In both works, the central node performs the neighbor discovery process. Similarly, the work did not focus on the energy consumption. In addition, the hidden nodes were assumed to be able to listen to the 'HELLO' messages broadcasted by the central node. Every node conducted the neighbor discovery in sensor networks, and hidden nodes could not be able to listen to the HELLO messages when sleeping. In [9], an algorithm for energy efficient node discovery is introduced. The approach uses the temporal patterns of happenstances and exploits the patterns to determine the duty cycling. Duty cycling is perceived as a sampling process and articulated as an optimization problem. Authors also used fortification-learning methods to perceive and dynamically change the times at which a sensor node should be aware, as it is likely to meet other nodes. In [6], a simple Aloha-like algorithm is proposed that supports synchronous node transmissions and the number of known nodes. The approach shows that the time for all the nodes to determine their corresponding neighbors is $\Theta(\ln n)$ in a perfect network, which led to an arbitrary number of nodes to communicate simultaneously. The fundamental problem of secure neighbor discovery is introduced in [12] and attempted to secure the network from different forms of attacks. The approach consists of a scalable key-distribution protocol that is secure in the absence of conspiring malicious nodes. The objectives were to present the secure neighbor discovery to protect the attacks of hidden nodes. The static network was proposed to secure the one-hop neighbor discovery process. However, the work did not focus on energy efficiency. All existing approaches tried to detect the hidden node, but did not factor in energy efficiency. Our proposed protocol particularly focuses on energy efficiency while detecting the hidden node in the neighborhood.

\section{DETECTING HIDDEN NODES}

We focus on the continuous neighbor discovery process. In our approach, each sensor node applies a simple approach in a coordinate effort to minimize energy consumption without increasing the required time to detect hidden nodes. In addition, we focus on avoiding the message collision.

Our approach consists of the following components:

\section{A. Hidden link participate inside a subdivision}

Our approach is to determine the hidden links when a new node joins the network. Subdivision nodes should detect the joining of new node in the network in order to continue the communication process. When new node joins that node releases a particular synchronization (SYN) message to all subdivision members, waking them up and periodically broadcasting a group of HELLO messages. This SYN message is dispersed over the already identified links of the subdivision. Thus, it is assured to be received in every subdivision node by having all the nodes wake up practically at the same time. For a short period, we can guarantee that every wireless link between the segment's members is detected.

\section{B. Hidden link participate outside a subdivision}

A random wake-up methodology is applied to reduce the option of iterating collisions between the HELLO messages of nodes in the same subdivision. In our approach, each node coordinates with its other neighborhood nodes during the wake-up periods to avert collisions and accelerate the discovery process of hidden nodes. Since the wake up time period is very small, and the time of forwarding the HELLO transmission time is even smaller. In this case, there is possibility that two nodes can be active at same time and initiate neighbor node recovery process. In our approach, we applied scheduling process so that there is marginal chance that two nodes should be active same time and initiate neighbor discovery process. During the scheduling process, the nodes need to be synchronized with each other and follow the schedule for the rest of communication time. Each receiving node chooses the some timeslots and receives the data during those timeslots. The time slot process is done without conflicting the schedule of other node. Therefore, we split the neighbor nodes into different subdivision groups, 
where node of each subdivision chooses its slot assigned to that group only.

\section{Neighbor Discovery Model}

The sensor node decides randomly, when to initiate the transmission of a HELLO message. If its message does not strike with another HELLO message, the node is referred to as a discovered node. We also, are able to determine the load and residual energy of each node after the node discovery process.

The load factor ' $L_{f}$ ' depends on the buffer state of the node that is estimated by using the equation (1).

$$
L_{f}=\left[\frac{B_{p}}{B_{\max }}\right]
$$

Where $B_{p}$ : The number of the Hello packets in the buffer and $B_{\max }$ : Maximum size of the buffer to accept the Hello message during the node discovery process. When the node discovery process is completed, we can determine the residual energy of the node. Thus residual energy ' $E_{r}{ }^{\prime}$ of each node can be calculated as

$$
E_{r}=E_{i}-\left(E_{t r}+E_{r e}\right)
$$

Where $E_{i}$ : Initial energy of node, $E_{r e}$ : Receiver's energy consumed for hidden node discovery process and $E_{t r}$ : transmitter's energy consumed for hidden node discovery process.

The objective is to determine the HELLO transmission frequency, and the duration of the neighbor discovery process.

\section{Algorithm 1: An Energy Efficient Hidden Node Detection Process}

1) Initialization of sensor nodes in a network. (Ns : sensor nodes)

2) Beginning of the simulation time (To).

3) Consider a hidden node in a network i.e $\mathrm{Nh}$

4) If (Nh $\in \mathrm{Ns}$ ), then

5) Nh broadcasts RREQ (root request) message forwarding to neighbor nodes $(\mathrm{Nn})$

6) Else if ( dest $==$ true), then

7) detects neighbor nodes and connection found

8) end if

9) detection is not found and unreachable

10) end if

11) consider state of nodes ( $\mathrm{Sn}$ : normal state; $\mathrm{Si}$ initial state) and probability "P1"

12) if ( $\mathrm{Sn}<<\mathrm{Si} \& \& \mathrm{Ns} \in \mathrm{Si})$, then

13) transmission of " HELLO" message takes place

14) else if (1-(1-P1)D $>=P)$, then

15) the function of DT(Discovery time) i.e TN(v )is calculated

16) end if

17) neighbor node $(\mathrm{Nn})$ responds to "HELLO" message through invoking

18) finalize the setup of joint wireless link
19) end if

In algorithm, line 1 shows the initialization process. Line- 2 sets the simulation time. Lines3-4 shows the number of nodes that belongs to the sensor network. From Lines5-10, neighbor nodes broadcast the message at 1-hop neighbor nodes. When the message is delivered and hidden nodes receive response, the availability of hidden nodes is presented. From lines 11-18 the energy consumption is illustrated based on the probability of hidden nodes and the discovery process. Finally, hidden nodes are allowed to join the networks. We take a simple example for the proposed algorithm. Let us assume that nodes in the initial state should remain an active until they enter into normal state. Let us consider a subdivision node in the normal state, where continuous neighbor discovery node is accomplished that determines the degree of its hidden neighbors. In our approach, we assume that distance of hidden node is 10 meters and number of hidden nodes are 2 . Thus, the hidden node wakes up after specified time and attempts to broadcast the message at the 1-hop neighborhood. This kind of process could help determine the availability of another hidden nodes in the network.

\section{SIMULATION SETUP AND PERFORMANCE ANALYSIS}

In order to evaluate the performance of our proposed energy efficient approach for the hidden node in the wireless multimedia sensor network, our approach was implemented using network simulator NS2. The network is designed to cover 800 X 800 square meters. We distributed 90 nodes in the network with homogenous capabilities. Each node has an initial 6 joules of energy. The simulation's objective is to determine the consumed energy for hidden nodes to improve the QoS. Furthermore, we also compared our approach with two other known existing approaches: Hidden-Node Avoidance Mechanism (H-NAMe) [13], and Hidden node avoidance for IEEE 802.15.4 (HNA-IEE) [14]. We demonstrated two scenarios, with and without hidden nodes. The simulation scenarios consist of 15 end nodes, which create flat topology. We set medium access layer to operate with the Non-Beacon enabled mode that applies un-slotted carrier sense multiple accesses with collision avoidance (CSMA/CA). Radio range of each sensor nodes under free-space propagation model is fixed to 45 meters. In first scenario, all end nodes that contribute in the network can listen to each other. The distance between each sensor node is set at 35 meters. In the second scenario, each sensor node can hear ten of the fifteen other end sensor nodes. This gives $50 \%$ probability to determine the hidden nodes without wasting energy. However, remaining 50\% nodes need to detect in the network. This scenario shows the near worst-case because and each collision could lead to a whole packet damage. End sensor node generates and transmits the acknowledged frame whose destination is head node (HN). Acknowledgement 
frame uses a constant application payload of 248 bits that involve 88 bits of the packet overhead, 48 network layer overhead, 64 bits medium access control overhead and 48 physical layer overhead. We generated the packets using interarrival time based on Rayleigh probability density function. In the first simulation experiment, the amount of received data traffic is referred as goodput that is observed as a utility of the generated data traffic for both scenarios (with and without hidden nodes). The network traffic shows an amount of data per time unit for non-acknowledgement frame that is either received or generated by the neighbor node. The load can be determined as

$$
N_{L}=N \times \beta \times F_{l}
$$

Where $N_{L}$ : Network load, $N$ : Number of nodes that generate the data, $\beta$ : Number of generated packets in unit time and $F_{l}$ : length of the frame that is equivalent to 248 bits.

The rest of parameters are explained in Table 1.

\begin{tabular}{|c|c|}
\hline PARAMTERS & VALUE \\
\hline Size of network & $800 \times 800$ square meters \\
\hline Number of nodes & 90 \\
\hline End nodes & 15 \\
\hline Queue-Capacity & 45 Packets \\
\hline Number of frames & 340frames \\
\hline $\begin{array}{l}\text { Maximum number of } \\
\text { retransmissions allowed }\end{array}$ & 03 \\
\hline Initial energy of node & 6 joules \\
\hline free-space propagation & 45 meters \\
\hline Size of Packets & 256 bytes \\
\hline Data Rate & 260 kilobytes/second \\
\hline Sensing Range of node & 35 meters \\
\hline Simulation time & 18 minutes \\
\hline Average Simulation Run & 08 \\
\hline Frame rate & $38 \mathrm{fps}$ \\
\hline Reliability & {$[0.78,0.92]$} \\
\hline Reporting rate & 3 packet/s \\
\hline Base station location & $(0,700)$ \\
\hline Transmitter Power & $12.7 \mathrm{~mW}$ \\
\hline Receiver Power & $13.6 \mathrm{~mW}$ \\
\hline Speed & $2-18 \mathrm{~m} / \mathrm{sec}$ \\
\hline
\end{tabular}

Based on simulation, we are interested in the following metrics.

- Successful delivered Packets

- End-to End delay

- Throughput with stationary nodes

A. Successful delivered packets

One of the penalties of the hidden node in WSNs is to drop the packets because of the collisions that destroy the contents' quality. Hence, the performance is highly degraded. We determine the ratio of successful packets as follows:

$$
P_{s}=\frac{P_{d}}{P_{g}} \times 100
$$

Where $P_{s}$ : Ratio of successful packets, $P_{d}$ : delivered packets and $P_{g}$ : Number of generated packets.

Figure 1 shows the results for the ratio of the successful packet delivery with and without hidden nodes. Without hidden nodes, the successful delivery is almost $96.9 \%$ with $180 \mathrm{~kb} / \mathrm{Sec}$ generated application traffic. Without hidden nodes, the network reaches its channel capacity; the numbers of generated packets get greater than the number of packets that can be transmitted. The packets that cannot be transmitted are lost. In the existence of the hidden nodes, packets are lost due to hidden node crashes and as traffic increases that lead to number of collisions. As a result, great number packets are lost.

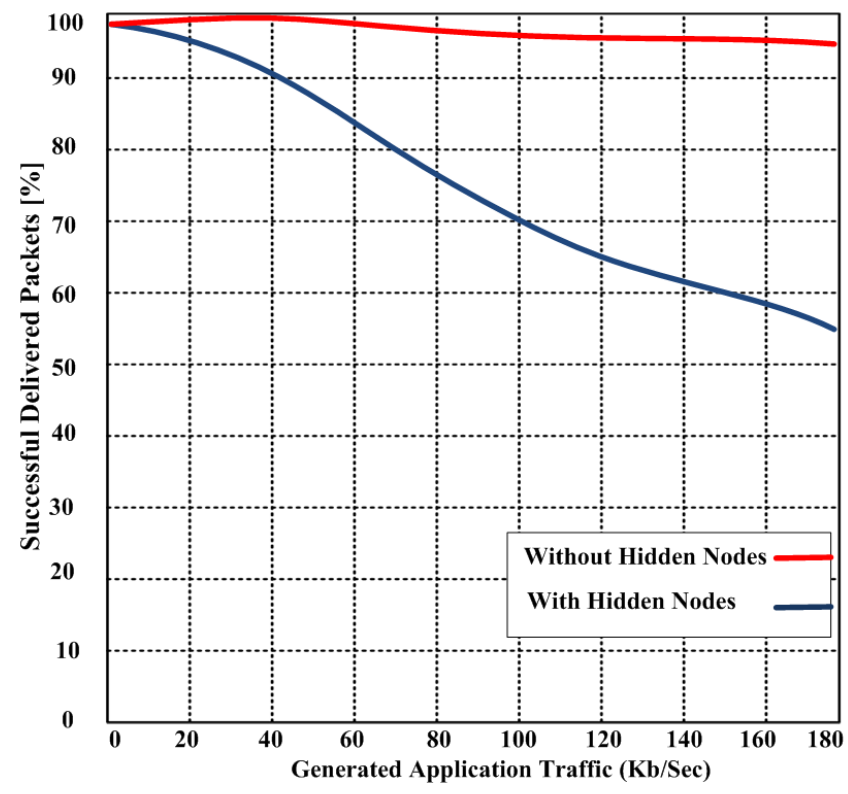

Figure 1: Successful delivery packets VS generated application traffic

B. End-to End delay

Packet delivery time shows the time that elapsed form its construction to the time when the packet is positively received by the destination node. Thus, this time is called End-to-End delay. Throughput performance of network depends on the end-to-end delay. If end-to-end delay is longer than usual time then, throughput performance is degraded. In Figure 2, we show the end-to- end delay of the network with and without hidden nodes. We observed that when the size of the application increases then end-to-end delay of the network is highly degraded in presence of the hidden nodes. This leads to the additional energy consumption and degradation in QoS. Furthermore, we show in Figure 3, the performance of network in presence of our proposed approach including the hidden and non-hidden nodes. The results demonstrate that our approach has substantially improved the performance of the network. The statistical data shows that our approach has minimum end-to-end delay up to $64 \mathrm{~kb} / \mathrm{sec}$. Once, the size of generated application increases then our approach has slightly higher end-to-end delay up to $139 \mathrm{~kb} / \mathrm{sec}$. 


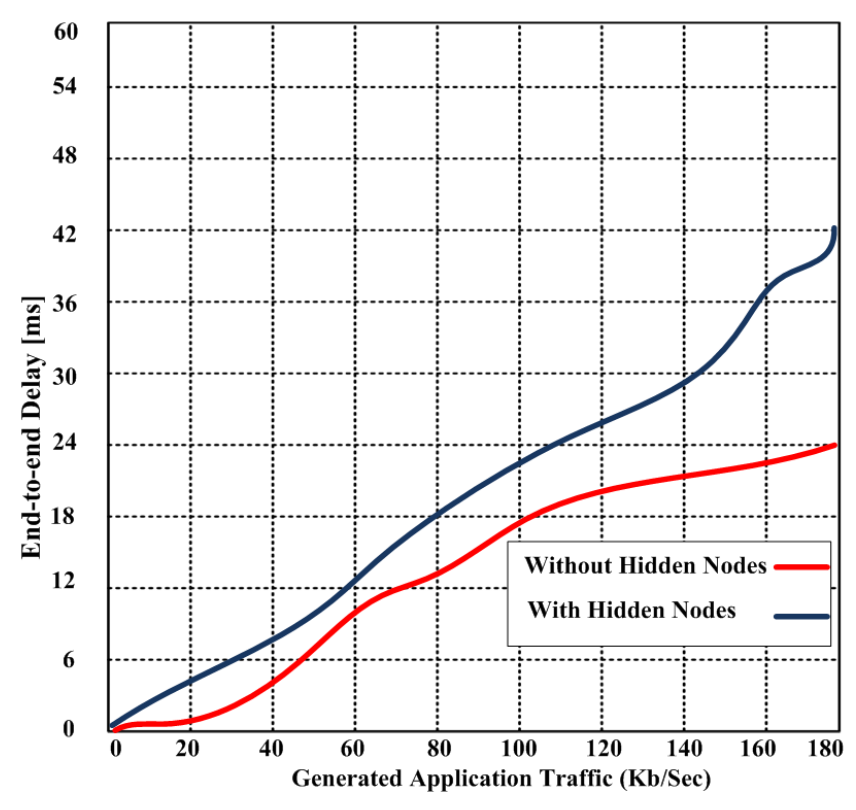

Figure 2: End-to-end delay VS generated application traffic

Furthermore, the trend of the results show that our approach has relatively similar end-to-end delay as obtained without hidden nodes. Based on the results, we conclude that our approach substantially decreased the latency and increased the energy efficiency that could lead improving the QoS in the network.

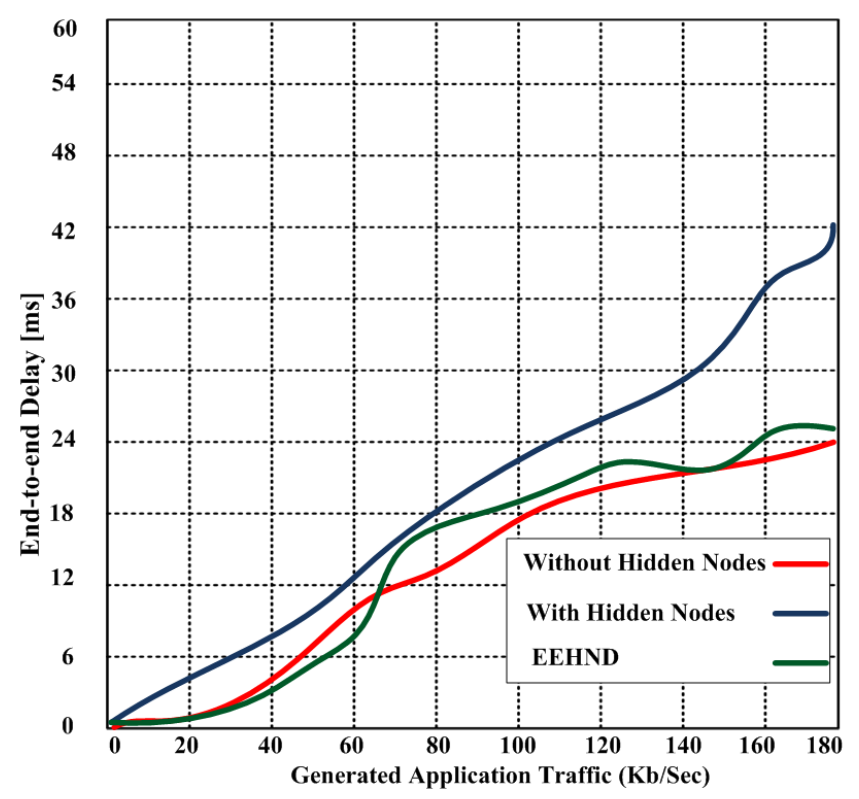

Figure 3: End-to-end delay of our proposed EEHND, with hidden nodes and without hidden nodes

C. Throughput with stationary nodes

The performance of EEHND is evaluated using $100 \%$ duty cycles with constant frame size of 256 data frame (including payload and data frame format). We conducated several runs to determine the network performance at the different loads.
Figures 4 and 5 show the throughput and success probability rate based on the experiments. The result demonstrates the performance of our proposed approach and other known approaches: H-NAMe) and (HNA-IEE).

The average value for throughput and probability success rate are calculated using $95 \%$ confidence interval for the sample size of 5000 packets at each given load. Based on the results, we observed that our proposed algorithm outperforms to other competing algorithms even at lower loads. For example, at a given offered load of $40 \%$, the success probability rate of our algorithm is greater than $36.8 \%$ than other competing algorithms. During the experiment scenario, two packets were retransmitted because of collision. If we deliberate one retransmission for each lost packet, the increase in the number of retransmission could be substantial in the case of the network without our proposed algorithm. This situation could lead to high-energy consumption.

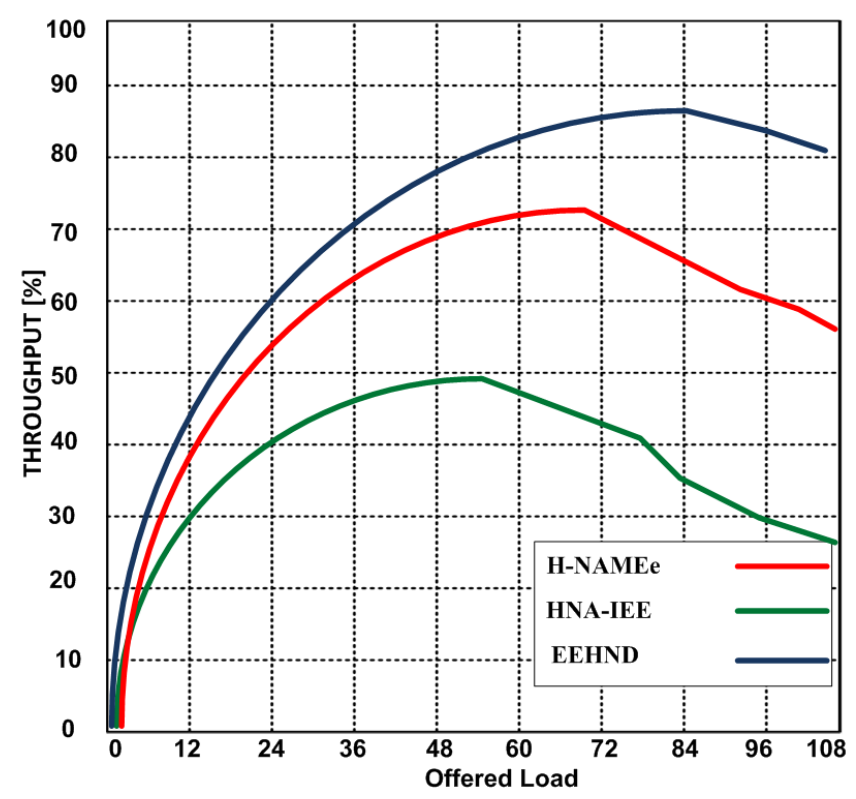

Figure 4. Throughput with different offered load

Considering higher loads, it is clear that our proposed EEHND increases the throughput up to $87.5 \%$ at $84 \%$ load. On the other hand, HNA-IEE increases the throughput up to $49.2 \%$ at the $54 \%$ load and H-NAMe increases up to 72.3 at the $70 \%$ load. We observed our algorithm provides $68 \%$ probability success at $108 \%$ load. While HNA-IEE and H-NAMe have $55.5 \%$ and $56.2 \%$ probability success respectively with same load.

Based on the results, we conclude that our proposed algorithm produced substantial progress in the network performance in terms of throughput and probability success rate. 


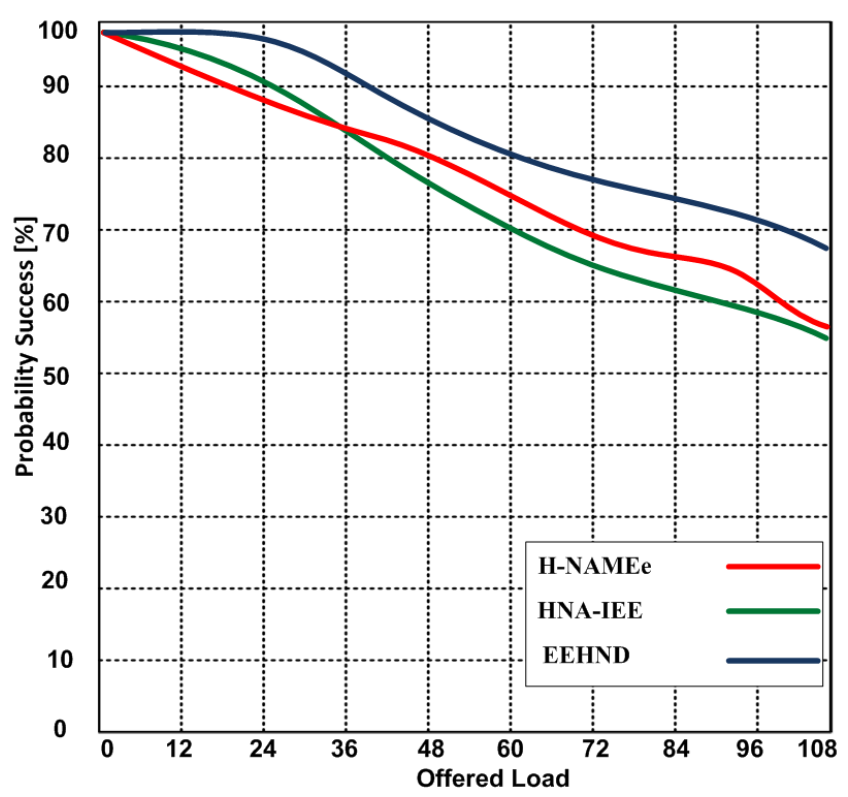

Figure 5. Probability success rate with different offered load

\section{CONCLUSION}

In this paper, an energy efficient hidden node detection algorithm is proposed for improving the Quality of Service of the wireless multimedia sensor networks. Our algorithm is based on continuous neighbor discovery process. Furthermore, we also included formulation to determine the load and residual energy of the nodes when completing the discovery process. We argue that continuous neighbor discovery is essential even if the sensor nodes are mobile. If the nodes in an associated subdivision work together on this task, hidden nodes are ensured to be discovered within a certain probability and a certain time period. Our simulation results demonstrate that our proposed EEHND algorithm improved the throughput performance of the network and reduced the end-to-end delay. Decrease in end-to-end delay can save the additional energy consumption. In addition, we compared the performance of our EEHND algorithm with HAN-IEE and NAMe approaches. The statistical data shows that our algorithm provides higher successful probability detection rate and throughput as compared with other approaches. The simulation results confirm that our proposed algorithm is capable of determining hidden nodes for WMSNs application. In future, we will measure the performance of our proposed algorithm in high scale wireless multimedia sensor networks and will apply hardware-testing process.

\section{REFERENCES}

R. Madan and S. Lall, "An energy-optimal algorithm for neighbor discovery in wireless sensor networks," Mobile Networks and Applications, vol. 11, pp. 317-326, 2006.

[2] A. Alanazi and K. Elleithy, "Real-Time QoS Routing Protocols in Wireless Multimedia Sensor Networks: Study and Analysis," Sensors, vol. 15, pp. 22209-22233, 2015.
[3] Z. Serhan and W. B. Diab, "Energy-efficient QoS routing in wireless multimedia sensor networks," in Advanced Information Networking and Applications (AINA), 2015 IEEE 29th International Conference on, 2015, pp. 223-230.

[4] K. Malarvizhi, M. Brindha, and M. Kumar, "Evaluation of energy efficient routing in wireless multimedia sensor networks," in Electronics and Communication Systems (ICECS), 2015 2nd International Conference on, 2015, pp. 1387-1391.

[5] D. J. Baker and A. Ephremides, "The architectural organization of a mobile radio network via a distributed algorithm," Communications, IEEE Transactions on, vol. 29, pp. 1694-1701, 1981.

[6] A. Russell, S. Vasudevan, B. Wang, W. Zeng, X. Chen, and W. Wei, "Neighbor Discovery in Wireless Networks with Multipacket Reception," 2014.

[7] R. Jurdak, P. Baldi, and C. V. Lopes, "Adaptive low power listening for wireless sensor networks," Mobile Computing, IEEE Transactions on, vol. 6, pp. 988-1004, 2007.

[8] P. Dutta and D. Culler, "Practical asynchronous neighbor discovery and rendezvous for mobile sensing applications," in Proceedings of the 6th ACM conference on Embedded network sensor systems, 2008, pp. 71-84.

[9] V. Dyo and C. Mascolo, "Efficient node discovery in mobile wireless sensor networks," in Distributed Computing in Sensor Systems, ed: Springer, 2008, pp. 478-485.

[10] A. Keshavarzian, E. Uysal-Biyikoglu, F. Herrmann, and A. Manjeshwar, "Energy-efficient link assessment in wireless sensor networks," in INFOCOM 2004. Twenty-third AnnualJoint Conference of the IEEE Computer and Communications Societies, 2004, pp. 1751-1761.

[11] S. A. Borbash, "Design considerations in wireless sensor networks," University of Maryland, 2004.

[12] S. Bagchi, S. Hariharan, and N. Shroff, "Secure neighbor discovery in wireless sensor networks," 2007.

[13] A. Koubâa, R. Severino, M. Alves, and E. Tovar, "H-NAMe: A hidden-node avoidance mechanism for wireless sensor networks," in 8th IFAC International Conference on Fieldbuses and Networks in Industrial and Embedded Systems, 2009, pp. 10-19.

[14] U. Pešović, J. Mohorko, S. Ranđić, and Ž. Čučej, "Hidden node avoidance mechanism for IEEE 802.15. 4 wireless sensor networks," Electronic Components and Materials, vol. 43, pp. 14$21,2013$.

Adwan Alanazi is pursuing his Doctorate in Computer Science and Engineering at the University of Bridgeport in Bridgeport, Connecticut, USA. His interests are wireless sensor networks, network routing, and mobile commutations.

Dr. Khaled Elleithy is the Associate Dean for Graduate Studies in the School of Engineering at the University of Bridgeport. He has research interests are in the areas of network security, mobile communications, and formal approaches for design and verification. He has published more than two hundred and fifty research papers in international journals and conferences in his areas of expertise.

Dr. Elleithy is the co-chair of the International Joint Conferences on Computer, Information, and Systems Sciences, and Engineering (CISSE). CISSE is the first Engineering/Computing and Systems Research E-Conference in the world to be completely conducted online in real-time via the Internet and was successfully running for six years. Dr. Elleithy is the editor or co-editor of 12 books published by Springer for advances on Innovations and Advanced Techniques in Systems, Computing Sciences and Software. 